Stud. Univ. Babeş-Bolyai Math. 65(2020), No. 3, 333-344

DOI: $10.24193 /$ subbmath.2020.3.02

\title{
Unit exchange elements in rings
}

\author{
Grigore Călugăreanu
}

\begin{abstract}
Replacing left principal ideals by cosets in the monoid $(R, \cdot)$ of a unital $\operatorname{ring} R$, we say that an element $a \in R$ is left unit exchange (or suitable) if there is an idempotent $e \in R$ such that $e-a \in U(R)\left(a-a^{2}\right)$ where $U(R)$ denotes the set of units. Unit-regular and clean elements are left (and right) unit suitable, and left (or right) unit suitable elements are exchange (suitable). The paper studies the multiple facets of this new notion.
\end{abstract}

Mathematics Subject Classification (2010): 16U99, 16U60, 13G99.

Keywords: Clean element, unit-regular element, exchange (suitable) element, unit suitable element, matrix rings.

\section{Introduction}

First recall that an element $a$ in a ring $R$ is clean if it is a sum of an idempotent and unit and strongly clean if these two commute. For an idempotent $e \in R$ we denote by $\bar{e}=1-e$ the complementary idempotent. The set of units of a (unital) ring $R$ is denoted by $U(R)$.

An element $a$ in a ring $R$ was defined as (see [5] for this numbering) left suitable (or exchange) by any of the following equivalent conditions:

(1) there is an idempotent $e \in R$ such that $e-a \in R\left(a-a^{2}\right)$.

(3) there is an idempotent $e \in R$ such that $e \in R a$ and $R e+R(1-a)=R$.

(4) there is an idempotent $e \in R$ such that $e \in R a$ and $1-e \in R(1-a)$. Replacing $R$ by $U(R)$, we introduce (similar to (1)) the following definition.

Definition 1.1. An element $a \in R$ is left unit suitable if there exists $e^{2}=e \in R$ such that $e-a \in U(R)\left(a-a^{2}\right)$. When we intend to emphasize the idempotent, $a$ will be called e-left unit suitable.

For an idempotent $e$ and a unit $u$ we consider the equation (called left eu-equation)

$$
P_{u, e}(x):=x^{2}-(1+u) x+e u=0 .
$$


It is readily seen that $x \in R$ is a solution of this equation iff

$$
\exists e^{2}=e \in R, \exists u \in U(R): u^{-1} e u-x=u^{-1}\left(x-x^{2}\right) .
$$

Therefore the left unit suitable elements in a ring are exactly the solutions of such eu-equations (more precisely, $e-a=u\left(a-a^{2}\right)$ is equivalent to $\left.P_{u^{-1}, u e u^{-1}}(a)=0\right)$. By computation $P_{u, e}(u+\bar{e})=0$, so

$\{$ clean elements $\} \subseteq\{$ left unit suitable elements $\} \subseteq\{$ suitable elements $\}$.

Examples in Section 3 will show that both inclusions may be proper.

However, it is easy to see that the left inclusion is equality in the following cases:

(i) $R$ has no zero divisors;

(ii) $R$ is a clean ring and in particular, is a matrix ring $\mathcal{M}_{n}(R)$ over any clean $\operatorname{ring} R$;

(iii) $R$ is Abelian and in particular commutative.

(iv) $R$ is Artinian and in particular finite.

Right unit suitable elements are defined symmetrically and similarly clean elements are right unit suitable and right unit suitable elements are suitable.

As an easy first example (which is largely generalized further), any square-zero element is trivially left and right unit suitable, since since $0-a=(-1)\left(a-a^{2}\right)$ holds whenever $a^{2}=0$.

In the first section, we give some useful characterizations for (left) unit suitable elements, for clean elements and for unit-regular elements, since such elements turn out to be left (or right) unit suitable. Since eu-equations are of degree two, some hints are given on the (possible) not clean solution.

The second section is devoted to results on left unit suitable $2 \times 2$ matrices. We show that over any commutative domain left unit suitable $2 \times 2$ matrices are also right unit suitable, we characterize left unit suitable zero lower row integral $2 \times 2$ matrices, trace 1 left (or right) unit suitable integral matrices via Diophantine equations and diagonal $2 \times 2$ left unit suitable matrices over any commutative domain. The matrix $\left[\begin{array}{cc}3 & 9 \\ 7 & -2\end{array}\right]$, already used in [1] as nil-clean but not clean, turns out to be suitable but not left (or right) unit suitable. Finally, a characterization of $2 \times 2$ unit-suitable matrices over any commutative domain is given, in connection again with unit-regular elements.

\section{Basic properties}

As already mentioned above, (left) suitable elements were defined in [5], by four equivalent conditions. Our definition corresponds to (1). Here is an equivalent definition corresponding to (4).

Proposition 2.1. An element $a \in R$ is left unit suitable iff there exist $e^{2}=e \in R$ and $b, c \in R$ such that $e=b a, 1-e=c(1-a)$ and $b-c \in U(R)$.

Proof. If $e-a=u\left(a-a^{2}\right)$ with $u \in U(R)$ then $e=[1+u(1-a)] a \in R a$ and $1-e=(1-u a)(1-a) \in R(1-a)$ and $b-c=1+u(1-a)-(1-u a)=u$. Conversely, 
if $e=b a, 1-e=c(1-a)$ and $b-c=u \in U(R)$ then $1-e=(b-u)(1-a)$ and so $1-b a=(b-u)(1-a)=b-u-b a+u a$ gives $b=1+u-u a$ and $e-a=(b-1) a=u(1-a) a$. Here $b=1+u-u a$ and $c=1-u a$.

A symmetric result holds for right unit suitable elements:

Proposition 2.2. An element $a \in R$ is right unit suitable iff there exist $e^{2}=e \in R$ and $b, c \in R$ such that $e=a b \in a R, 1-e=(1-a) c \in(1-a) R$ and $b-c \in U(R)$.

Here $b=1+u-a u, c=1-a u$ and again $b-c=u$.

Corollary 2.3. Left (or right) unit suitable elements have the "complement property", that is, if $\alpha$ is left (or right) unit suitable, so is $1-\alpha$.

In [7], another class of rings, intermediate between clean and suitable rings is introduced, under the name of weakly clean rings (and elements). Recall that weakly clean elements do not have the "complement property" (see Remark 4.7 (ii) in [8]), so these are different elements compared to left (or right) unit suitable elements (by the previous corollary).

"For any $a, b \in R, 1-a b$ is a unit iff $1-b a$ is a unit" is known as Jacobson's lemma for units.

Since this lemma fails for clean elements and for suitable elements but holds for (unit) regular elements, we could ask whether it holds or fails for left (and/or right) unit suitable elements. Actually it fails: in [4] an example of clean (and so also left and right unit suitable) matrix $C D \in \mathcal{M}_{2}(\mathbf{Z})$ is given, for which $D C$ is not suitable (and so nor left or right unit suitable). It remains to use the previous corollary.

The set of left unit suitable elements in a ring also includes the unit regular elements. More, we can prove the following characterization (with above notations)

Proposition 2.4. A left unit suitable element a is unit regular iff, with the notations in the previous proposition, $c^{2}=c$ and $a c=0$.

Proof. Suppose $a=a u a$ with $u \in U(R)$. Then $u a$ and so $c=1-u a$ are both idempotents and $a c=0$. Take $b=c+u$. Then $b a-a=u(1-a) a$ and $b a b a=b a$ shows that $a$ is left unit suitable (one can also check $1-b a=c(1-a)$ ). Conversely, assume $c^{2}=c, a c=0,1-b a=c(1-a)$ and $b-c=u \in U(R)$. By left multiplication with $a$ we get $a-a b a=a c-a c a=0$ so $a b a=a$. Hence $a u a=a(b-c) a=a b a=a$, as desired.

A symmetric result holds for right unit suitable elements.

In particular, unit regular elements are left and right unit suitable and so elements which are both left and right suitable need not be clean (see the example after Theorem 3.3, in the next section).

An elementary trick, more or less always used in the context of exchange rings, is the following: for a ring $R$ and elements $a, e \in R$, if $e \in R a$ is an idempotent, an element $b \in R$ can be chosen such that $e=b a$ and $b a b=b$. Note that such an element $b$ is regular.

Recall (see Introduction) that an element $a \in R$ was called left suitable (or exchange) if there is an idempotent $e \in R$ such that $e \in R a$ and $1-e \in R(1-a)$. 
Using the previous observation, (regular) elements $b, c \in R$ can be chosen such that $e=b a, b a b=b, 1-e=c(1-a)$ and $c(1-a) c=c$.

Coming back to our initial definition, we could consider elements $a \in R$ such that the elements $b, c$ above can be chosen with $b-c \in U(R)$.

This restriction is too strong because it is easy to prove the following characterization (already noticed in [2])

Proposition 2.5. An element $a$ in a ring $R$ is clean iff there exist $b, c \in R$ such that $b a b=b, c(1-a) c=c, 1-b a=c(1-a)$ and $b-c \in U(R)$.

Notice that if only one from the conditions $b a b=b, c(1-a) c=c$ holds, the statement above is no longer valid. An example is given after Theorem 3.3.

Rephrasing, a left unit suitable element a is clean iff for the regular elements $b, c$ emphasized above with $b a b=b$ and $c(1-a) c=c, b-c$ is a unit.

Remark 2.6. Since we already noticed that $x=u+\bar{e}$ is a solution of the eu-equation $P_{u, e}(x)=0$, we could wonder when this degree two polynomial factors into two degree one polynomials. Denoting by $a$ the second solution it is easy to show that $P_{u, e}(x)=x^{2}-(1+u) x+e u=(x-u-\bar{e})(x-a)$ iff $(u+\bar{e}-x) a=e(u-x)$. If $u e=e u$ then $a=e$ is a clean solution.

\section{Unit suitable $2 \times 2$ matrices}

Recall that a ring $R$ is Dedekind finite (DF for short) if, for every $a, b \in R, a b=1$ implies $b a=1$. A ring $R$ is stably finite if the matrix rings $\mathcal{M}_{n}(R)$ are Dedekind finite for all natural numbers $n$.

We first point out some simple but general results.

Proposition 3.1. (a) In any DF ring, the 0-left unit suitable elements are clean.

(b) In any DF ring, the 0-left unit suitable elements are also right unit suitable.

(c) For any positive integer $n$, and any stably finite ring $k, 0_{n}$-left unit suitable matrices in $\mathcal{M}_{n}(k)$ are clean.

(d) In any DF ring, the 1-left unit suitable elements are units and so clean.

(e) In any DF ring, the 1-left unit suitable elements are also right unit suitable.

(f) For any positive integer $n$, and any stably finite ring $k, I_{n}$-left unit suitable matrices in $\mathcal{M}_{n}(k)$ are clean.

Proof. (a) First notice that $e-a=u\left(a-a^{2}\right)$ is equivalent to $1-e=(1-u a)(1-a)$. Taking $e=0$ yields $1=(1-u a)(1-a)$ and if the ring is DF, $1-a$ is a unit. Hence $a$ is clean.

(b), (c) follow from (a).

(d) We just notice that $1=a+u\left(a-a^{2}\right)$ is now equivalent to $1=[1+u(1-a)] a$, so $a$ is a unit.

(e), (f) follow from (d).

If $k$ is a commutative domain, then $E \in \mathcal{M}_{2}(k)$ is an idempotent iff $E=0_{2}, E=I_{2}$ or $\operatorname{det}(E)=0$ and $\operatorname{Tr}(A)=1$. 
Theorem 3.2. Left unit suitable $2 \times 2$ matrices over a commutative domain $k$ are also right unit suitable.

Proof. Suppose $A \in \mathcal{M}_{2}(k)$ is left unit suitable, i.e. $E=A+U\left(A-A^{2}\right)$ with a unit $U$ and an idempotent $E$.

If $E=0_{2}$ or $E=I_{2}$ the result follows from Proposition 3.1 (b) and (e), respectively.

In the remaining case, assume $\operatorname{det}(E)=0$ and $\operatorname{Tr}(E)=1$. Letting

$$
F=A+\left(A-A^{2}\right) U \text {, }
$$

we have

$$
\operatorname{Tr}(F)=\operatorname{Tr}(A)+\operatorname{Tr}\left(\left(A-A^{2}\right) U\right)=\operatorname{Tr}(A)+\operatorname{Tr}\left(U\left(A-A^{2}\right)\right)=\operatorname{Tr}(E)=1 .
$$

Moreover, $\operatorname{det}(E)=0$ gives $\operatorname{det}\left(I_{2}+U\left(I_{2}-A\right)\right) \operatorname{det}(A)=0$, hence

$$
\operatorname{det}\left(I_{2}+U\left(I_{2}-A\right)\right)=0 \text { or } \operatorname{det}(A)=0 .
$$

Notice that for any $2 \times 2$ matrix $M$ over a commutative ring,

$$
\operatorname{det}\left(I_{2}+M\right)=\operatorname{det}(M)+\operatorname{Tr}(M)+1 .
$$

Therefore,

$$
\begin{aligned}
\operatorname{det}\left[I_{2}+U\left(I_{2}-A\right)\right] & =\operatorname{det}\left[U\left(I_{2}-A\right)\right]+\operatorname{Tr}\left[U\left(I_{2}-A\right)\right]+1 \\
& =\operatorname{det}\left[\left(I_{2}-A\right) U\right]+\operatorname{Tr}\left[\left(I_{2}-A\right) U\right]+1 \\
& =\operatorname{det}\left[I_{2}+\left(I_{2}-A\right) U\right]
\end{aligned}
$$

and so $\operatorname{det}\left[I_{2}+U\left(I_{2}-A\right)\right]=0$ iff $\operatorname{det}\left[I_{2}+\left(I_{2}-A\right) U\right]=0$. It follows that

$$
\operatorname{det}\left(I_{2}+\left(I_{2}-A\right) U\right)=0 \quad \text { or } \operatorname{det}(A)=0,
$$

hence $\operatorname{det}(F)=\operatorname{det}\left(I_{2}+\left(I_{2}-A\right) U\right) \operatorname{det}(A)=0$. This shows that $\operatorname{Tr}(F)=1$ and $\operatorname{det}(F)=0$, proving that $F$ is an idempotent. Hence $A$ is right unit suitable (in this case, with respect to the same unit).

In contrast with suitable elements, it is unlikely that the set of left unit suitable rings and the set of right unit suitable coincide. As seen above, the always good source of examples, $\mathcal{M}_{2}(\mathbf{Z})$, cannot be used when searching for a left unit suitable element which is not right unit suitable.

Since $\mathbf{Z}$ is not exchange (and so nor clean), in searching for unit suitable elements which are not clean, it is worth trying with $\mathcal{M}_{2}(\mathbf{Z})$. In searching for left unit suitable elements which are not clean, or suitable elements which are not left unit suitable we first prove the following

Theorem 3.3. For a matrix $A=\left[\begin{array}{ll}a & b \\ 0 & 0\end{array}\right] \in \mathcal{M}_{2}(\mathbf{Z})$, the following are equivalent:

1. $A$ is unit-regular or clean in $\mathcal{M}_{2}(\mathbf{Z})$.

2. $A$ is left unit suitable in $\mathcal{M}_{2}(\mathbf{Z})$.

3. $A$ is suitable in $\mathcal{M}_{2}(\mathbf{Z})$.

4. $(a, b)$ is a unimodular row or $a \in\{0,2\}$. 
Proof. $1 \Rightarrow 2 \Rightarrow 3$ follow from earlier results in the paper (and hold for any ring element).

$3 \Rightarrow 4$ : Suppose that $A$ is suitable and $\operatorname{gcd}(a, b)=n>1$ (i.e. $(a, b)$ is not unimodular). By the suitable property, $E=R A$ and $I_{2}-E=S\left(I_{2}-A\right)$ for some $R, S, E \in \mathcal{M}_{2}(\mathbf{Z})$ with $E^{2}=E$. Since $A \in n \mathcal{M}_{2}(\mathbf{Z})$, it follows that $E \in n \mathcal{M}_{2}(\mathbf{Z})$, hence $E=0$ as $E=E^{2}$.

Hence $I_{2}=S\left(I_{2}-A\right)$ so that $I_{2}-A$ is a unit. The determinant of this matrix is $1-a$. Hence $1-a \in\{ \pm 1\}$, i.e. $a \in\{0,2\}$.

$4 \Rightarrow 1$ : If $(a, b)$ is unimodular then $A$ is clearly unit-regular, and if $a \in\{0,2\}$ then $I_{2}-A$ is a unit so that $A$ is clean.

Recall from [3] (Theorem 4.7) that if $(a, b)$ is a reduced unimodular row (i.e. $|a| \geq 2|b|$ and $a, b$ generate the unit ideal $),\left[\begin{array}{ll}a & b \\ 0 & 0\end{array}\right]$ is clean iff $a \equiv \pm 1(\bmod b)$.

Therefore, again according to [3], the reduced unimodular rows $(12,5),(13,5)$, $(12,7),(13,8),(17,5),(16,7),(18,5),(17,7)$ yield not clean unit-regular matrices which are left unit suitable.

Example 3.4. For $A=\left[\begin{array}{cc}12 & 5 \\ 0 & 0\end{array}\right], U=\left[\begin{array}{cc}19 & 8 \\ 7 & 3\end{array}\right]$ and $E=\left[\begin{array}{cc}8 & -8 \\ 7 & -7\end{array}\right]$ one checks the eu-equation

$$
X^{2}-\left(I_{2}+U\right) X+E U=0_{2} .
$$

This example also suits as a left unit suitable element with

$$
C\left(I_{2}-A\right) C=C=\left[\begin{array}{cc}
-35 & -15 \\
84 & 36
\end{array}\right]
$$

(that is, regular $C$ ) but

$$
B A B=\left[\begin{array}{cc}
-32 & 32 \\
77 & -77
\end{array}\right] \neq\left[\begin{array}{cc}
-32 & -23 \\
77 & 55
\end{array}\right]=B
$$

(so not regular $B$ ). Here

$$
B-C=\left[\begin{array}{cc}
3 & -8 \\
-7 & 19
\end{array}\right]
$$

is a unit, $A U^{-1} A=A$ and both

$$
B A=F=\left[\begin{array}{cc}
-384 & -160 \\
924 & 385
\end{array}\right]
$$

(here $\left.F-A=U^{-1}\left(A-A^{2}\right)\right), A B=\left[\begin{array}{cc}1 & -1 \\ 0 & 0\end{array}\right]$ are idempotents.

Remark 3.5. The nice idempotent obtained in the previous example,

$$
A B=\left[\begin{array}{cc}
1 & -1 \\
0 & 0
\end{array}\right]
$$

is not specific. It is the result for any matrix

$$
A=\left[\begin{array}{ll}
a & b \\
0 & 0
\end{array}\right]
$$


with coprime $a, b$. As seen in the previous proof,

$$
U=A+\left[\begin{array}{ll}
z & u_{22} \\
z & u_{22}
\end{array}\right]
$$

with $a u_{22}-b z=1$. Then

$$
U^{-1}=\left[\begin{array}{cc}
u_{22} & -u_{22} \\
-z & z
\end{array}\right]+\left[\begin{array}{cc}
0 & -b \\
0 & a
\end{array}\right]=S+T
$$

and indeed

$$
A S=\left[\begin{array}{cc}
1 & -1 \\
0 & 0
\end{array}\right] \text { and } A T=0_{2}
$$

Hence

$$
A B=A+A U^{-1}\left(I_{2}-A\right)=A U^{-1}=A S=\left[\begin{array}{cc}
1 & -1 \\
0 & 0
\end{array}\right] .
$$

Obviously $A B$ is idempotent and in general

$$
B A B=\left[\begin{array}{cc}
r & s \\
m & n
\end{array}\right] A B=\left[\begin{array}{cc}
r & s \\
m & n
\end{array}\right]\left[\begin{array}{cc}
1 & -1 \\
0 & 0
\end{array}\right]=\left[\begin{array}{cc}
r & -s \\
m & -n
\end{array}\right] \neq\left[\begin{array}{cc}
r & s \\
m & n
\end{array}\right]=B
$$

unless $n=s=0$.

Since unipotent elements (i.e. sums $1+t$ with nilpotent $t$ ) are special units in rings, we could ask whether (left) unipotent suitable elements are not clean. First notice (e.g. from the proof of the previous theorem), that in general there is no uniqueness for the unit in the definition of the (left) unit suitable elements. So a better rephrased question would be: if for a (left) unit suitable element, the unit can be chosen as an unipotent, is the element clean? The answer is no as shows the next example.

Example 3.6. Take the second unimodular row in the list above (from [3]). For the matrix $A=\left[\begin{array}{cc}13 & 5 \\ 0 & 0\end{array}\right], U=\left[\begin{array}{cc}5 & 2 \\ -8 & -3\end{array}\right]$ and $E=\left[\begin{array}{cc}-7 & 7 \\ -8 & 8\end{array}\right]$ one checks the euequation

$$
X^{2}-\left(I_{2}+U\right) X+E U=0_{2} .
$$

Therefore $A$ is left unipotent suitable but not clean (it is readily checked that unipotents in $\mathcal{M}_{2}(\mathbf{Z})$ are the matrices of trace $=2$ and determinant $=1$ ).

As mentioned above, the same matrix satisfies the eu-equation with $U=\left[\begin{array}{cc}18 & 7 \\ 5 & 2\end{array}\right]$ and $E=\left[\begin{array}{ll}6 & -6 \\ 5 & -5\end{array}\right]$ where $U$ is no more unipotent.

Similar results (on zero upper row, or columns) may be obtained using conjugation by $\left[\begin{array}{ll}0 & 1 \\ 1 & 0\end{array}\right]$ or transposes.

The ring $\mathcal{M}_{2}(\mathbf{Z})$ contains many suitable elements that are not left unit suitable. Matrices with trace 1 are definitely one example, but there are also other classes that are easier to characterize. One of those classes are the diagonal matrices. Below is given a characterization of suitable and left unit suitable diagonal matrices. 
Lemma 3.7. Let $A=\left[\begin{array}{ll}a & 0 \\ 0 & b\end{array}\right]$ be a $2 \times 2$ diagonal matrix over a commutative domain $k$ and let $d \in k$. The following are equivalent:

1. There exists $X \in \mathcal{M}_{2}(k)$ with $\operatorname{det}(X)=d$ such that $E=A+X\left(A-A^{2}\right)$ is a nontrivial idempotent (i.e. $E \neq 0_{2}, I_{2}$ ).

2. There exists $t \in k$ such that $t(a-b)-d\left(a-a^{2}\right)=1$.

Proof. $1 \Rightarrow 2$ : Suppose that $E=A+X\left(A-A^{2}\right)$ is a nontrivial idempotent and $\operatorname{det}(X)=d$. Write $\alpha=a-a^{2}, \beta=b-b^{2}$ and $X=\left[\begin{array}{cc}x & y \\ z & w\end{array}\right]$. Then

$$
E=\left[\begin{array}{cc}
a+x \alpha & y \beta \\
z \alpha & b+w \beta
\end{array}\right]
$$

and since $E$ is nontrivial $\operatorname{Tr}(E)=1$, hence

$$
a+b+x \alpha+w \beta=1 \text {. }
$$

If $b=0$ then this equation gives $a+x \alpha=1$, whence $a \in U(k)$. Hence, taking $t=(1+d \alpha) a^{-1}$ we get

$$
t(a-b)-d \alpha=t a-d \alpha=(1+d \alpha)-d \alpha=1,
$$

which proves the claim. Similarly if $b=1$ then (3.1) gives $a+x \alpha=0$.

Hence $1+x(1-a) a=0$ which implies $1-a \in U(k)$. Hence taking $t=(1+d \alpha)(a-1)^{-1}$ we get $t(a-b)-d \alpha=t(a-1)-d \alpha=(1+d \alpha)-d \alpha=1$, as desired. Thus we may assume $b \neq 0,1$ and so $\beta \neq 0$.

The determinant condition $\operatorname{det}(E)=0$ gives

$$
a b+a w \beta+b x \alpha+\alpha \beta d=0 .
$$

Now (3.1) and (3.2) together give

$$
b=b(a+b+x \alpha+w \beta)-(a b+a w \beta+b x \alpha+\alpha \beta d)=b^{2}+(b-a) w \beta-\alpha \beta d,
$$

hence $\beta=(b-a) w \beta-\alpha \beta d$. Cancelling $\beta$ we obtain $1=(b-a) w-\alpha d$ and so $t=-w$ fulfills the desired condition.

$2 \Rightarrow 1$ : Take $t$ with $t(a-b)-d\left(a-a^{2}\right)=1$. Letting

$$
X=\left[\begin{array}{cc}
t+d(a+b-1) & -1 \\
t(t+d(a+b-a)) & -t
\end{array}\right]
$$

it is easy to see that $\operatorname{det}(X)=d$ and $E=A+X\left(A-A^{2}\right)$ satisfies $\operatorname{Tr}(E)=1$ and $\operatorname{det}(E)=0$, which proves the claim.

Theorem 3.8. For a $2 \times 2$ matrix $A=\left[\begin{array}{ll}a & 0 \\ 0 & b\end{array}\right]$ over a commutative domain $k$, the following are equivalent:

1. A is suitable. $t, s \in k$.

2. Either $A$ is a unit or $I_{2}-A$ is a unit or $t(a-b)+s\left(a-a^{2}\right)=1$ for some 
Proof. $1 \Rightarrow 2$ : Let $E=A+X\left(A-A^{2}\right)$ with idempotent $E$ and $X \in \mathcal{M}_{2}(k)$. If $E=I_{2}$ then $A$ is a unit, and if $E=0_{2}$ then $I_{2}-A=X A\left(I_{2}-A\right)+I_{2}$, so that $I_{2}-A$ is a unit. Therefore we may assume that $E$ is a nontrivial idempotent. By the previous lemma we obtain $t \in k$ such that $t(a-b)-\operatorname{det}(X)\left(a-a^{2}\right)=1$. Hence $t$ and $s=-\operatorname{det}(X)$ satisfy the required condition.

$2 \Rightarrow 1$ : If $A$ or $I_{2}-A$ is invertible then there is nothing to prove. Thus let $t(a-b)+s\left(a-a^{2}\right)=1$ with $t, s \in k$. Using again the previous lemma we get a matrix $X$ with $\operatorname{det}(X)=-s$ such that $E=A+X\left(A-A^{2}\right)$ is a nontrivial idempotent, as desired.

Theorem 3.9. For a $2 \times 2$ matrix $A=\left[\begin{array}{ll}a & 0 \\ 0 & b\end{array}\right]$ over a commutative domain $k$, the following are equivalent:

1. A is left unit suitable.

2. $A$ is clean.

3. Either $A$ is a unit or $I_{2}-A$ is a unit or $t(a-b)+s\left(a-a^{2}\right)=1$ for some $t \in k$ and $s \in U(k)$.

Proof. $2 \Rightarrow 1$ is clear

$1 \Rightarrow 3$ : Suppose $A$ is left unit suitable, i.e. $E=A+U\left(A-A^{2}\right)$ with idempotent $E$ and unit $U$. As before, if $E=I_{2}$ then $A$ is a unit, and if $E=0_{2}$ then $I_{2}-A$ is a unit. Therefore we may assume $E$ is nontrivial. Hence by Lemma 3.7 there exists $t \in k$ with $t(a-b)-\operatorname{det}(U)\left(a-a^{2}\right)=1$, so that $t$ and $s=-\operatorname{det}(U)$ satisfy the desired condition.

$3 \Rightarrow 2$ : If $A$ or $I_{2}-A$ is invertible there is nothing to prove. Thus let

$$
t(a-b)+s\left(a-a^{2}\right)=1
$$

for some $t \in k$ and $s \in U(k)$. We can check directly that

$$
E=\left[\begin{array}{cc}
a-s^{-1} t & 1+t\left(a+b-1-s^{-1} t\right) \\
s^{-1} & 1-a+s^{-1} t
\end{array}\right]
$$

is an idempotent (with $\operatorname{Tr}(E)=1$ and $\operatorname{det}(E)=0$ ) and $U=A-E$ is a unit (with $\left.\operatorname{det}(U)=s^{-1}\right)$.

Example 3.10. Let $a=2$ and $b=-3$. Then, taking $t=1$ and $s=2$ we get

$$
t(a-b)+s\left(a-a^{2}\right)=1 \cdot 5+2 \cdot(-2)=1,
$$

so that $A=\left[\begin{array}{cc}2 & 0 \\ 0 & -3\end{array}\right]$ is suitable in $\mathcal{M}_{2}(\mathbf{Z})$ by Theorem 3.8. However, the equation $t \cdot 5+s \cdot(-2)=1$ clearly has no solution in $\mathbf{Z}$ if $s \in U(\mathbf{Z})$, hence $A$ is not left unit suitable in $\mathcal{M}_{2}(\mathbf{Z})$ by Theorem 3.9 .

Next we prove another result which connects unit-suitable $2 \times 2$ matrices with unit-regular ones.

Theorem 3.11. For a $2 \times 2$ matrix $A$ over a commutative domain $k$, the following are equivalent:

1. A is unit-suitable. 
2. Either $A$ is unit-regular or $I_{2}-A$ is unit-regular or there exists a unit $U$ such that the pairs $(\operatorname{Tr}(U), \operatorname{det}(U A))$ and $(\operatorname{Tr}(U A)-1, \operatorname{det}(U)(\operatorname{Tr}(A)-1))$ have the same sum and the same product.

Proof. For any $2 \times 2$ matrix, Cayley-Hamilton's theorem gives

$$
A-A^{2}=\operatorname{det}(A) I_{2}-(\operatorname{Tr}(A)-1) A .
$$

Hence, a matrix $A$ is (left) unit-suitable iff there is a unit $U$ such that

$$
E=\left(I_{2}+U\left(I_{2}-A\right)\right) A=A+\operatorname{det}(A) U-(\operatorname{Tr}(A)-1) U A
$$

is an idempotent.

As mentioned in the proof of Theorem 3.2, if $E=0_{2}$ then $I_{2}-A$ is a unit and if $E=I_{2}$ then $A$ is a unit (and units are unit-regular). In the remaining case, assume $\operatorname{Tr}(E)=1$ and $\operatorname{det}(E)=0$.

Notice that equivalently $\operatorname{det}(E)=0$ gives $\operatorname{det}\left(I_{2}+U\left(I_{2}-A\right)\right) \operatorname{det}(A)=0$, hence

$$
\operatorname{det}\left(I_{2}+U\left(I_{2}-A\right)\right)=0 \quad \text { or } \operatorname{det}(A)=0 .
$$

By (3.3), $\operatorname{Tr}(E)=1$ is equivalent to $\operatorname{det}(A) \operatorname{Tr}(U)=(\operatorname{Tr}(A)-1)(\operatorname{Tr}(U A)-1)$. Case 1. If $\operatorname{det}(A)=0$ then $\operatorname{Tr}(A)=1$ or $\operatorname{Tr}(U A)=1$ and since

$$
\operatorname{det}(U A)=\operatorname{det}(U) \operatorname{det}(A)=0,
$$

$A$ or $U A$ is a (nontrivial) idempotent. As well-known, in both cases $A$ is unit-regular. Case 2. If $\operatorname{det}(A) \neq 0$ then $\operatorname{det}\left(I_{2}+U\left(I_{2}-A\right)\right)=0$. Notice that for any $2 \times 2$ matrix $B$

$$
\operatorname{det}\left(I_{2}+B\right)=1+\operatorname{Tr}(B)+\operatorname{det}(B),
$$

so the previous condition amounts to $1+\operatorname{Tr}\left(U\left(I_{2}-A\right)\right)+\operatorname{det}\left(U\left(I_{2}-A\right)\right)=0$.

Equivalently,

$$
1+\operatorname{Tr}(U)-\operatorname{Tr}(U A)+\operatorname{det}(U)[1-\operatorname{Tr}(A)+\operatorname{det}(A)]=0
$$

or

$$
1+\operatorname{Tr}(U)+\operatorname{det}(U A)=\operatorname{Tr}(U A)+\operatorname{det}(U)(\operatorname{Tr}(A)-1) .
$$

Multiplying $\operatorname{det}(A) \operatorname{Tr}(U)=(\operatorname{Tr}(A)-1)(\operatorname{Tr}(U A)-1)$ by $\operatorname{det}(U)$ shows that the pairs $(\operatorname{Tr}(U), \operatorname{det}(U A))$ and $(\operatorname{Tr}(U A)-1, \operatorname{det}(U)(\operatorname{Tr}(A)-1))$ have the same sum and the same product.

Corollary 3.12. Let $A$ be a $2 \times 2$ matrix over a commutative $\operatorname{domain} k$, and $\operatorname{det}(A)=0$. Then $A$ is unit-suitable iff either $A$ is unit-regular or $I_{2}-A$ is unit-regular.

Proof. One way follows from the previous proof, and the converse follows from Corollary 2.3, since unit-regular elements are unit-suitable.

For integral matrices we can say more.

Corollary 3.13. Let $A$ be an integral $2 \times 2$ matrix. Then $A$ is unit-suitable iff either $A$ is unit-regular or $I_{2}-A$ is unit-regular or there exists a unit $U$ such that

$$
\operatorname{Tr}(U A)=1+\operatorname{det}(U A), \quad \operatorname{Tr}(U)=\operatorname{det}(U)(\operatorname{Tr}(A)-1) .
$$


Proof. Indeed, since the pairs in the previous theorem are roots of the same degree two (solvable) equation we have

$$
\{\operatorname{Tr}(U), \operatorname{det}(U A)\}=\{\operatorname{Tr}(U A)-1, \operatorname{det}(U)(\operatorname{Tr}(A)-1)\} .
$$

Therefore

$$
\operatorname{Tr}(U A)=1+\operatorname{det}(U A), \quad \operatorname{Tr}(U)=\operatorname{det}(U)(\operatorname{Tr}(A)-1)
$$

or

$$
\operatorname{Tr}(U A)=1+\operatorname{Tr}(U), \quad \operatorname{det}(U A)=\operatorname{det}(U)(\operatorname{Tr}(A)-1) .
$$

In the second case, we can show that $I_{2}-A$ is a unit-regular element.

Recall that we are working in the hypothesis of Case 2 (the proof of the previous theorem), that is,

$$
\operatorname{det}\left(I_{2}+U\left(I_{2}-A\right)\right)=0
$$

Since

$$
\operatorname{Tr}\left(I_{2}+U\left(I_{2}-A\right)\right)=2+\operatorname{Tr}(U)-\operatorname{Tr}(U A)=1
$$

it follows that $E:=I_{2}+U\left(I_{2}-A\right)$ is an idempotent. Hence

$$
I_{2}-A=-U^{-1}\left(I_{2}-E\right)
$$

is unit-regular (indeed, an element $b \in R$ is unit-regular iff there are a unit $u$ and an idempotent $e$ such that $b=u e)$.

Trace 1 left (or right) unit suitable integral matrices can be characterized via Diophantine equations. We just mention the following

Proposition 3.14. (i) A trace 1, $2 \times 2$ integral matrix $A=\left[\begin{array}{cc}a+1 & b \\ c & -a\end{array}\right]$ with $b \neq 0$ is (left or right) unit suitable iff

$$
b x^{2}-(2 a+1) x y-c y^{2}+(1+\operatorname{det}(A)) y+b=0
$$

and

$$
b \text { divides } 1+\operatorname{det}(A)-(2 a+1) x-c y
$$

or else

$$
b x^{2}-(2 a+1) x y-c y^{2}+(1-\operatorname{det}(A)) y-b=0
$$

and

$$
b \text { divides } 1-\operatorname{det}(A)-(2 a+1) x-c y \quad \text { (4) }
$$

(ii) The matrix $C=\left[\begin{array}{cc}3 & 9 \\ -7 & -2\end{array}\right]$ is a $2 \times 2$ suitable (exchange) matrix which is not left nor right unit suitable in $\mathcal{M}_{2}(\mathbf{Z})$.

Remark 3.15. This shows that $C$, our example in [1], can be used to improve its initial purpose: this is a nil-clean matrix which is not unit suitable (not only not clean). As for now, the problem of finding an example of nil-clean element which is not suitable (exchange) remains open. 


\section{References}

[1] Andrica, D., Călugăreanu, G., A nil-clean $2 \times 2$ matrix over integers which is not clean, J. of Algebra and its Applications, 13(6)(2014), 9 pages.

[2] Călugăreanu, G., Split-extensions of Exchange Rings: a direct proof, J. of Algebra and Applications, 8(5)(2009), 629-632.

[3] Khurana, D., Lam, T.Y., Clean matrices and unit-regular matrices, J. of Algebra, 280(2004), 683-698.

[4] Lam, T.Y., Nielsen, P., Jacobson's lemma for Drazin inverses, Ring Theory and its Appl., Contemporary Math., 609(2014), 185-196.

[5] Nicholson, W.K., Lifting idempotents and exchange rings, Trans. Amer. Math. Soc., 229(1977), 269-278.

[6] Nicholson, W.K., On exchange rings, Comm. Algebra, 25(6)(1997), 1917-1918.

[7] Šter, J., Corner rings of a clean ring need not be clean, Comm. Algebra, 40(5)(2012), 1595-1604.

[8] Šter, J., Weakly clean rings, J. of Algebra, 401(2014), 1-12.

Grigore Călugăreanu

Babeş-Bolyai University,

Faculty of Mathematics and Computer Science,

Str. Kogălniceanu, 1,

400084 Cluj-Napoca, Romania

e-mail: calu@math.ubbcluj.ro 